\title{
Pentavalent vaccination in rural Kenya: coverage and geographical accessibility to health facilities using data from a community demographic and health surveillance system in Kilifi County.
}

Morris Ogero ( $\square$ ogeromorris@gmail.com )

Aga Khan University Hospital https://orcid.org/0000-0003-0117-6289

James Orwa

Aga Khan University

Rachael Odhiambo

Aga Khan University

Felix Agoi

Aga Khan University

Adelaide Lusambili

Aga Khan University

Jerim Obure

Aga Khan University

Marleen Temmerman

Aga Khan University

Stanley Luchters

Aga Khan University

Anthony Ngugi

anthony.ngugi@aku.edu

Research

Keywords: pentavalent vaccination, health facilities accessibility

Posted Date: September 9th, 2020

DOl: https://doi.org/10.21203/rs.3.rs-72783/v1

License: (c) (1) This work is licensed under a Creative Commons Attribution 4.0 International License.

Read Full License 


\section{Abstract}

Background There is substantial evidence that immunization is one of the most significant and costeffective pillars of preventive and promotive health interventions. Effective childhood immunization coverage is thus essential in stemming persistent childhood illnesses. The main indicator of performance of the immunisation programme is the third dose of diphtheria-tetanus-pertussis (DTP3) vaccine for children, because it mirrors the completeness of a child's immunisation schedule. Spatial access to a health facility, especially in SSA countries, is a significant determinant of DTP3 vaccination coverage as the vaccine is mainly administered during routine immunisation schedules at health facilities. Rural areas and densely populated informal settlements are most affected by poor access to healthcare services. We therefore sought to determine vaccination coverage of DTP3, estimate the travel time to health facilities offering immunisation services, and explore of its effect on immunisation coverage in one of the predominantly rural counties in the coast of Kenya.

Methods Coordinates of health facilities, information on land cover, digital elevation model, and road network were used to compute spatial accessibility to immunising health facilities for eligible children within Kaloleni-Rabai Community Health Demographic Surveillance System (HDSS). To explore the effect of the travel-time on DTP3 coverage, we fitted a hierarchical multivariable model adjusting for other apriori identified confounding factors.

Results Spatial access to health facilities that offer immunization services significantly affected DTP3 coverage with travel times of more than one hour to a health facility significantly associated with reduced odds of receiving DTP3 vaccine (AOR= $0.84(95 \% \mathrm{Cl} 0.74-0.94)$.

Conclusion Increased travel time is a significant barrier to the uptake of facility-delivered immunizations in this rural community. To improve immunisation coverage, local health authorities and policy makers in remote settings can use high resolution maps to identify areas where distance and travel time may impede achievement of high immunizations coverage and identify appropriate interventions. These could include improving the road network, establishing new health centres and/or stepping up health outreach activities that include vaccinations in hard to reach areas within the county.

\section{Introduction}

One of the global Sustainable Development Goals (SDG) targets towards reducing childhood mortality from preventable deaths is ensuring universal vaccination coverage[1]. Estimates put lives saved through immunization at 2-3 million per year [2], which is substantial evidence that immunization is one of the most significant and yet cost-effective pillars of preventive and promotive health interventions [3]. The establishment of the World Health Organisation's Expanded Program of Immunization (EPI)- whose mandate is to ensure equitable access to routine immunization services, resulted in introduction of more vaccines and better global coverage. The coverage of initial core vaccines (BCG, DTP, Polio, and measles vaccine) increased from $5 \%$ in 1974 to over $86 \%$ in 2018 [4, 5]. Despite the impressive global statistics, 
there are substantial inter- and intra-country heterogeneities of vaccine coverage resulting in approximately 19.4 million unimmunised children in 2018 . The majority of these children are from subSaharan countries $[4,6]$ where the mortality rate from vaccine - preventable diseases for the under-fives remains among the highest in the world [7].

The third dose of diphtheria-tetanus-pertussis (DTP3) vaccine for children is an important indicator of assessing performance of the immunisation programme because it mirrors the completeness of a child's immunisation schedule [5]. For this reason, the Global Vaccine Action Plan (GVAP) set a dual target for DTP3 at $90 \%$ in national coverage and $80 \%$ for other administrative units by year 2020 [8]. According to the Global Alliance for Vaccines and Immunizations (GAVI), Kenya national estimates of DTP3 coverage was $81 \%$ in 2018 [9]. However, there is potential masking of spatial heterogenicities especially in rural areas or areas of low coverage as a result of averaging across regions. This might allow pockets of preventable infectious diseases to persist [10], which could act as foci for potential future outbreaks.

Spatial access to a health facility, especially in sub-Saharan Africa (SSA) countries, is a significant determinant of DTP3 vaccination coverage as the vaccine is mainly administered during routine immunisation schedules at health facilities [11-13]. Studies have shown that rural areas [14,15] and densely populated informal settlements $[16,17]$ are most affected by poor access to healthcare services. Although factors that influence access to immunisation services have been studied extensively in a broader sense [12,18-22], the local context within communities, which to a larger extent determines how these factors interact, has not been explored. Furthermore, the role of spatial access to primary health services is poorly described in Kenya. In this study we sought to determine vaccination coverage of DTP3, estimate the travel time to health facilities offering immunisation services, and explore its effect on immunisation coverage in one of the predominantly rural counties (Kilifi) in the coast of Kenya.

\section{Methods}

\section{Study area}

This study utilized data from the Kaloleni-Rabai Community Health Demographic Surveillance System (HDSS). This HDSS cohort is centred around Mariakani township and covers 112 villages spanning 10 Community Health Units (CHU) of Kaloleni and Rabai sub-counties, Kilifi County, Kenya (Fig. 1), three of which are peri urban. This cohort has been followed up semi-annually since 2017 and six rounds of data collection had been completed by the end of 2019. New individuals can enter the cohort by either birth or in-migration, while cohort members can exit by either out-migration or death. A detailed profile of this cohort has been described elsewhere [23]. Each member of the cohort is assigned unique identifier at entry, which is used to longitudinally track the individual.

\section{Data Collection}

For each round of data collection, a trained community health volunteer (CHV) visited the longitudinally tracked households and interviewed the mother or caretaker of the child who provided the following data: vaccination data (based on child's vaccination card or on maternal recall if card is unavailable), and 
demographic information, reproductive, maternal and child-health data, child orphan status, school attendance among children, social determinants of disease, nutritional data, vital events (births, migration, and deaths). DPT3 immunization data were captured for all children 14 weeks - 11 months of age. Global positioning System (GPS) coordinates of the households was also collected. Pre-configured Open Data Kit (ODK) installed in electronic tablets was used for data collection and upon completion of the interview, data were reviewed for completeness and synced to a central server. Further data screening was performed by a data manager for any errors (omissions, and inconsistencies) and the feedback sent to $\mathrm{CHV}$ for verification. The whole process of data collection was supervised and coordinated by field officers and the ministry of health public health personnel.

In each round of data collection, the data were analysed, and the audit reports per $\mathrm{CHU}$ shared with $\mathrm{CHVs}$ who in return coordinated the dissemination sessions with the community, where they discussed key areas of active feedback including vaccination status among others.

\section{Assembling of geospatial data necessary for the estimation of spatial accessibility}

To compute spatial access to health facilities, the following information was assembled; coordinates of health facilities, land cover, digital elevation model, road network, and barriers. While the households of interest were within Kaloleni and Rabai Sub-counties of Kilifi county, and with the assumption that the nearest health facility might be in the neighbouring counties especially for households along the borders of the neighbouring Sub-counties, we confined the analysis of the spatial accessibility to include immunising health facilities, digital elevation model (DEM), land cover, and road network from the counties neighboring the study area as shown in Fig. 2.

\section{Health facilities}

Since healthcare facilities are critical in the delivery of vaccines, we obtained a list of all facilities that offer immunization services within the study area and the neighbouring administrative areas from the Kenya master health facility list [24] and the Kenya health information system [25]. We merged facilities from these two sources, eliminated duplicates and obtained their GPS coordinates, which we validated against the recently geocoded master database of all health facilities in sub-Saharan Africa [26]. Furthermore, we ensured that the resultant heath facilities were within the settlement and not on waterbodies by checking their coordinates using Google Earth.

\section{Road Network}

Data for road network were assembled from OpenStreetMaps (OSM) and Google Map Maker (GMM). Duplicates and short sections of roads disconnected from the main network were removed. As done elsewhere [27, 28], we classified roads into 4 categories; primary (class A \& B) roads that mainly connect international borders, secondary (class C \& D) roads that feed into primary roads or connected to major towns, county (class E) roads that feed into secondary roads and also connects smaller towns or market centers, and rural (class $U$ ) these are unclassified roads that connects rural areas. These roads were 
assigned different speeds depending on the probable mode of transport as follows; primary and secondary roads whose mode of transport were vehicular were assigned a speed of $80 \mathrm{~km} / \mathrm{h}$ and $50 \mathrm{~km} / \mathrm{h}$ respectively. County roads with bicycling as a mode of transport were assigned $11 \mathrm{~km} / \mathrm{h}$ while rural roads were assigned $5 \mathrm{~km} / \mathrm{h}$ based on the similar studies in Kenya $[28,29]$.

\section{Digital Elevation model \& Land cover}

We obtained data for land cover and digital elevation model (DEM) at a spatial resolution of $30 \mathrm{~m}$ from the Regional Centre for Mapping of Resources for Development (RCMRD)[30]. This is the centre responsible for disseminating open geospatial datasets for Eastern and Southern Africa. Land cover for the study area consisted of 9 categories which we assigned walking speed based on previous studies [28, $29,31]$; tree cover $(4 \mathrm{~km} / \mathrm{h})$, shrubs cover $(5 \mathrm{~km} / \mathrm{h})$, grassland $(5 \mathrm{~km} / \mathrm{h})$, cropland $(2 \mathrm{~km} / \mathrm{h})$, aquatic vegetation $(0.01 \mathrm{~km} / \mathrm{h})$, sparse vegetation $(2 \mathrm{~km} / \mathrm{h})$, bare areas $(5 \mathrm{~km} / \mathrm{h})$, built up areas $(5 \mathrm{~km} / \mathrm{h})$, and open water $(0.01 \mathrm{~km} / \mathrm{h})$. Walking and bicycling speeds were further adjusted accordingly based on the topography derived from DEM. This correction used Tobler's equation [32] that linked walking and bicycling speeds with the slope of the terrain.

$$
W=6 * \exp \left(-3.5 \text { abs }\left[\tan \left(\frac{S}{57.296}\right)+0.05\right]\right),
$$

where $W$, is the speed calculated and $S$ is the slope in degrees

Land covers and the DEM showing different elevations of the study area are provided in the supplementary file 1.

\section{Estimation Of Travel Time Using Geographic Accessibility Model}

Methods for estimating geographical accessibility have been developed over time namely; travel time model [27], network analysis [33], and gravity model [34]. In this study we used travel time model because it has been recommended by WHO as a suitable method of modeling healthcare accessibility [35], and also because it takes into considerations other key aspects of accessing care such as terrain and land cover surfaces [36] .

We used AccessMod (version 5)[37] to model geographical accessibility. The software uses Manhattan distance method in determining cumulatively the time needed to cross the contiguous cells using the least cost path from settlement to immunising health facilities. Therefore, to estimate travel time, we first generated a travel impedance raster surface by merging land cover, elevation, and road network. To each contiguous cell of the resultant raster layer, we assigned travel speeds accordingly as described earlier. Lastly, we combined the location of the immunising health facilities to the rasterized layer and estimated the time in minutes needed to travel to the nearest facility at $30 \mathrm{~m}$ spatial resolution. For further analyses, we extracted the travel time for each household's geographical coordinates from the generated raster file. The obtained travel time was then distributed to children within a given household. Maps of travel time to 
the nearest immunising health facility and the average time per household were plotted in QGIS (version 3.12).

\section{Statistical Analyses}

We used Bayesian hierarchical logistics regression model to explore the effect of spatial accessibility on DTP3 coverage. Community health units (CHU), and round of data collection were used as random effects. To stabilize computations, we used weakly informative priors which also serve to bind the estimates within the acceptable ranges [38]. We specified four chains each with 5000 iterations half of which were used to warmup the sampler and was discarded before estimations were made. Convergence of the model was determined by examining trace plots of the model. We adjusted for confounding due to socio-demographic and other health system factors available in the dataset (see Fig. 3). In keeping with the previous studies investigating the effect of travel time [29], we grouped travel time into two; less than 1-hour, and more than 1-hour travel seeking health service. To compare differences between two groups we used an independent $t$-test statistical technique and the results were interpreted using $p$-value at the significance level of $a=0.05$. The results from the multivariable model were reported as odd ratios (OR), and $95 \%$ credible intervals. Significance of odds ratios was assumed if the $95 \%$ credible intervals excluded one. All analyses were performed using R Version 3.4.3.

\section{Results}

\section{Characteristics and the vaccination status of the children in the cohort}

We included data for 4,442 children aged 14 weeks to 11 months in the cohort. The study population was distributed in $10 \mathrm{CHUs}$ with a median number of children per CHU being 303 (IQR = 181-404). Majority of the children were female 2,261(51\%) and most of them (93.1\%) slept under treated mosquito nets. The median travel time to a health facility was $41(\mathrm{IQR}=18-65)$ minutes and a total of $1266(28.5 \%)$ children lived more than one-hour travel to immunising health facilities. Demographic characteristics were not appreciably different between vaccinated and unvaccinated children as shown in Table 1. We observed that coverage of DTP3 in the cohort improved over time (rounds of data collection) from $62 \%$ in January to June 2017 (round 1) to 93\% in July to December 2019 (round 6) (see Fig. 4). The overall coverage was $3435(77.3 \%)$ and this coverage varied across CHUs from 70.9-88.8\% (see Fig. 5). Within the study area there was a total of 32 immunising health facilities. Figure 6 provides a visual representation of the travel time to the nearest immunising health facilities using the combined modes of transport: walking/cycling and motorized transport. The data suggested that children who lived 30 minutes of travel from the immunising health facilities had a DTP3 coverage of $82.6 \%$ compared to a coverage ratio of $62.1 \%$ in children with longer travel times (more than 2 hours from the immunising health facilities). In comparing travel time between type of residence, we observed that the median travel time was 17 minutes (IQR 831 ) and 54 (IQR 33-75) minutes in rural and peri-urban settlement respectively ( $p$.value $<0.001)$. 
Table 1

Characteristics of the HDSS children $<11$ months eligible for DTP3 vaccination

\begin{tabular}{|c|c|c|c|}
\hline Indicator & $\begin{array}{l}\text { DPT3 vaccinated ( } \\
=3435)\end{array}$ & $\begin{array}{l}\text { DPT3 not vaccinated } \\
(n=1007)\end{array}$ & $\begin{array}{l}\text { Overall }(n= \\
\text { 4442) }\end{array}$ \\
\hline Gender (Female) & $1743(50.7 \%)$ & $518(51.4 \%)$ & $\begin{array}{l}2,261 \\
(51.0 \%)\end{array}$ \\
\hline Peri-urban area of residence & $1,189(34.6 \%)$ & $223(22.1 \%)$ & $\begin{array}{l}1,412 \\
(31.8 \%)\end{array}$ \\
\hline Use safe water & $1331(38.7 \%)$ & $328(32.6 \%)$ & $\begin{array}{l}1,659 \\
(37.3 \%)\end{array}$ \\
\hline Treats drinking water & $1959(57.0 \%)$ & $519(51.5 \%)$ & $\begin{array}{l}2,478 \\
(55.8 \%)\end{array}$ \\
\hline $\begin{array}{l}\text { Hand-washing facility in a } \\
\text { household }\end{array}$ & $1174(34.2 \%)$ & $355(35.3 \%)$ & $\begin{array}{l}1,529 \\
(34.4 \%)\end{array}$ \\
\hline $\begin{array}{l}\text { Ownership of latrine/toilet by a } \\
\text { household }\end{array}$ & $2143(62.4 \%)$ & 576 (57.2\%) & $\begin{array}{l}2,719 \\
(61.2 \%)\end{array}$ \\
\hline Has a birth certificate & $159(4.6 \%)$ & $62(6.2 \%)$ & $221(5.0 \%)$ \\
\hline Is an orphan & $67(2.0 \%)$ & $26(2.6 \%)$ & $93(2.1 \%)$ \\
\hline $\begin{array}{l}\text { Sleep under mosquito-treated } \\
\text { net }\end{array}$ & $3247(94.5 \%)$ & $889(88.3 \%)$ & $\begin{array}{l}4,136 \\
(93.1 \%)\end{array}$ \\
\hline More than $1 \mathrm{hr}$ travel time & $959(27.9 \%)$ & $375(37.2 \%)$ & $\begin{array}{l}1,334 \\
(30.1 \%)\end{array}$ \\
\hline
\end{tabular}

\section{Factors influencing DTP3 vaccination coverage.}

Results from the multivariable model are shown in Table 2. Increased mean travel time to immunising health facility was associated with reduced odds of being vaccinated. Precisely, the results suggest that travel time of more than one hour to health facility significantly reduced the likelihood of DTP3 vaccination by approximately $16 \%$ after adjusting for other factors (adjusted odds ratio $=0.84(95 \% \mathrm{Cl}$ 0.74-0.94)). Other factors included in the model namely; sleeping under treated mosquito net, children given vitamin A supplements, and ownership of birth certificates were associated with an increased likelihood of a child receiving DTP3 vaccine. WASH indicators namely hand washing, treating drinking water, and use of latrine were not associated with DTP3 vaccination. Furthermore, other factors such as child sex and type of settlement (rural or peri-urban) were not significant predictors of DTP3 vaccination (see Table 2). 
Table 2

Multivariate model for factors influencing DTP3 vaccination coverage

\begin{tabular}{|ll|}
\hline Characteristics & Adjusted odds ratio $(95 \% \mathrm{Cl})$ \\
\hline Urban area of residence (Urban) & $1.02(0.73-1.44)$ \\
\hline Treats drinking water & $1.09(0.97-1.21)$ \\
\hline Hand-washing facility in a household & $0.82(0.74-0.92)$ * \\
\hline Ownership of latrine/toilet by a household & $1.04(0.93-1.17)$ \\
\hline Has a birth certificate & $1.27(1.00-1.61)$ * \\
\hline Is an orphan & $0.80(0.59-1.10)$ \\
\hline Child sex (Male) & $1.01(0.93-1.11)$ \\
\hline Sleep under mosquito-treated net & $1.36(1.13-1.65)$ * \\
\hline Given vitamin A supplements & $6.41(5.82-7.07)$ * \\
\hline More than 1hr travel time & $0.84(0.75-0.94)$ * \\
\hline *denotes statistical significance at 5\% significance level \\
\hline
\end{tabular}

\section{Discussion}

In this study, we sought to estimate the coverage of DTP3 vaccination and the influence of travel time to vaccination centres in one of the predominantly rural counties in the coast of Kenya using data from a community demographic and health surveillance system. Recognising that DTP3 vaccine is primarily administered during routine immunisation at health centres, we hypothesised that spatial accessibility was a key factor in determining DTP3 vaccination uptake. The data from the surveillance system suggested that over three-quarters of the eligible children had received DTP3 vaccine. While this immunisation coverage is commendable, it was however below the GVAP goal of achieving $90 \%$ by year 2020 [8]. We observed that the mean travel time to immunising facilities was $44.9(S D=31.2)$ minutes, assuming a composite mode of transport of walking/cycling and motorised transport. This varied significantly by place of residence (rural and peri-urban). We also noted that $30.1 \%$ of children lived more than one hour of travel from a health facility, this is far below the Kenyan policy recommendation that states that $90 \%$ of the population should live within one hour of walking speed from a health facility that offers immunisation services [39]. Travel times of more than one hour to a health facility was significantly associated with reduced odds of receiving DTP3 vaccination (AOR $=0.84$ ( $95 \% \mathrm{Cl} 0.74-0.94)$ and travel times of more than two hours was associated with a DTP3 coverage ratio of $62.1 \%$ which is below the set target. 
Previous studies on the barriers of accessing healthcare $[40,41]$ have shown that time required to travel to a healthcare facility, particularly in sub-Saharan Africa, is the main barrier to accessing healthcare. In this study, we used a combination of walking, cycling and motorised transport to estimate the travel times to health facilities that offered immunisation services. The effect of spatial access on the immunisation coverage has been explored by previous studies $[11-13,42,43]$ and they have shown that travel time influences the uptake of child vaccination. In addition to spatial accessibility, a number of studies have also shown that child birth order, wealth quintiles, and exposure to media content positively influence immunisation coverage, especially in low and middle income countries [44-46]. However, in this study, we did not explore these factors as we were only interested in estimating effects of spatial accessibility to immunisation coverage with a view of making recommendations to the local government to increase and space out evenly the number of health facilities that offer immunisation services in the area. We however assumed that these factors would be applicable in this context.

Involvement of community health workers/community health volunteers in childhood vaccination has been shown to be both efficient and cost-effective in expanding immunisation coverage and improving reporting systems, especially in hard to reach areas [47]. Our data demonstrated a marked improvement of DTP3 coverage over time since the inception of the community surveillance system whose data was used in this study (Fig. 6). This further demonstrates the value addition to immunization coverage that CHVs' involvement in child immunization services can offer. In this study, the use of CHVs, coupled with integrated audit and feedback activities embedded in the community by the CHVs, improved the overall adoption of recommended immunization practices over time [48]. We posit that engaging CHVs in regular data collection in the households provided for increased contact with household members, which afforded them opportunities for enhanced health education and promotion, including tracing defaulters of essential health services such as vaccinations. We also noted marked differences in immunisation coverage in different $\mathrm{CHUs}$, this could be due to group dynamics and subtle geographical differences within the study area. Other factors such as education levels, incentives and capacity building could also have contributed to differences in performance of the CHUs [49]. Factors such as ignorance on need for immunisations, missing return dates for next immunization schedule, fear of adverse events following immunisation, negative attitude of health care providers and missed opportunities for vaccination have also been highlighted as factor which are contributing to low vaccination coverage [50].

\section{Strength And Limitations}

This study had several limitations. First, travel time estimations did not consider factors that might affect travel speed especially in rainy season, frequency of transport services, and traffic flow. Other nuances that are likely to affect care-seeking behaviour such as variation of the quality of healthcare services [51], health professionals' strikes[52], and stock-outs could not be adjusted for in the model. To determine DTP3 coverage, we used data from vaccination card and mother's recall in the absence of vaccination card. The inclusion of maternal recall potentially introduced recall bias. Lastly, it is possible that the list of immunising facilities used in this study did not include make-shift facilities also provide immunisation services. 


\section{Conclusions}

This study aimed to determine DTP3 vaccination coverage in one of the predominantly rural counties in the coast of Kenya using data from a community demographic and health surveillance system. We found that DTP3 coverage was significantly affected by spatial access to health facilities that offer immunization services with travel times of more than one hour to a health facility significantly associated with reduced odds of receiving DTP3 vaccine. In order to improve immunisation coverage, especially for DTP3, high resolution map of estimated travel time to the nearest healthcare facility could be used by local health authorities, policy makers and relevant stakeholders to identify places for intervention in order to improve physical accessibility to healthcare facilities in the community. Interventions could include improving the road network, establishing new health centres and/or stepping up health outreach activities that include vaccinations in hard to reach areas within the county.

\section{List Of Abbreviations}

BCG - Bacille Calmette -Guerin

$\mathrm{CHU}$ - Community Health Unit

$\mathrm{CHV}$ - Community Health Volunteer

DTP3 - Diphtheria-Tetanus-Pertussis

EPI - Expanded Programme of Immunization

GAVI - Global Alliance for Vaccines and Immunizations

GVAP - Global Vaccine Action Plan

GPS - Global Positioning System

GMM - Google Map Maker

ODK - Open Data KitOSM - OpenStreetMaps

SSA - Sub-Saharan Africa

WHO - World Health Organization

\section{Declarations}

Consent for publication:

Permission was granted from the caretaker or mother to publish this work 


\section{Competing interests:}

The authors declare that they have no competing interests.

\section{Funding}

Aga Khan Foundation Canada and the Government of Canada \#7540280 have supported this research as part of the Access to Quality Care for Extending and Strengthening Health Services (AQCESS) project.

\section{Author contribution}

MO, JO, and AN contributed in the conceptualisation, design, data analysis and interpretation. RO, JO, FA, $A L, M T, S L$ contributed to the revision of the manuscript and intellectual rigor of the content. All authors read and approved the final manuscript.

\section{Acknowledgements:}

We thank the Ministry of Health who gave permission for this work to be developed.

\section{References}

1. United Nations, Sustainable development goals report 2016. 2016: UN.

2. World Health Organization, 2018 assessment report of the Global Vaccine Action Plan: strategic advisory group of experts on immunization. 2018, World Health Organization.

3. Nzioki, J.M., et al., Community health worker interventions are key to optimal infant immunization coverage, evidence from a pretest-posttest experiment in Mwingi, Kenya. Pan African Medical Journal, 2017. 28(1).

4. World health Organization. Immunization coverage. 2019 [cited Feb 2020; Available from: https://www.who.int/news-room/fact-sheets/detail/immunization-coverage.

5. Subaiya, S., et al., Global routine vaccination coverage, 2014. Morbidity and Mortality Weekly Report, 2015. 64(44): p. 1252-1255.

6. WHO/UNICEF, Global Immunization Data: Global Immunization Coverage. 2018.

7. UNICEF, Progress for children: A report card on immunization. 2005: UNICEF.

8. World Health Organization, Global vaccine action plan 2011-2020. 2013.

9. Global Alliance for Vaccines and Immunizations(GAVI). Programmes \& Impact. 2020 [cited 2020; Available from: https://www.gavi.org/programmes-impact/country-hub/africa/kenya.

10. Utazi, C.E., et al., High resolution age-structured mapping of childhood vaccination coverage in low and middle income countries. Vaccine, 2018. 36(12): p. 1583-1591.

11. Vyas, P., D. Kim, and A. Adams, Understanding Spatial and Contextual Factors Influencing Intraregional Differences in Child Vaccination Coverage in Bangladesh. Asia Pacific Journal of Public Health, 2019. 31(1): p. 51-60. 
12. Okwaraji, Y.B., et al., The association between travel time to health facilities and childhood vaccine coverage in rural Ethiopia. A community based cross sectional study. BMC public health, 2012. 12(1): p. 476.

13. Devkota, S. and B. Panda, Childhood immunization and access to health care: evidence from Nepal. Asia Pacific Journal of Public Health, 2016. 28(2): p. 167-177.

14. Favin, M., et al., Why children are not vaccinated: a review of the grey literature. International health, 2012. 4(4): p. 229-238.

15. Rainey, J.J., et al., Reasons related to non-vaccination and under-vaccination of children in low and middle income countries: findings from a systematic review of the published literature, 1999-2009. Vaccine, 2011. 29(46): p. 8215-8221.

16. Ahmed, S., et al., Impact of traffic variability on geographic accessibility to $24 / 7$ emergency healthcare for the urban poor: A GIS study in Dhaka, Bangladesh. PloS one, 2019. 14(9).

17. Panciera, R., et al., The influence of travel time on emergency obstetric care seeking behavior in the urban poor of Bangladesh: a GIS study. BMC Pregnancy Childbirth, 2016. 16(1): p. 240.

18. Gwatkin, D. and G. Deveshwar-Bahl, Immunization coverage inequalities. An overview of socioeconomic and gender differentials in developing countries. The World Bank, 2001.

19. Wiysonge, C.S., et al., Individual and contextual factors associated with low childhood immunisation coverage in sub-Saharan Africa: a multilevel analysis. PLoS One, 2012. 7(5).

20. Egondi, T., et al., Determinants of immunization inequality among urban poor children: evidence from Nairobi's informal settlements. International journal for equity in health, 2015. 14(1): p. 24.

21. Hajizadeh, M., Socioeconomic inequalities in child vaccination in low/middle-income countries: what accounts for the differences? J Epidemiol Community Health, 2018. 72(8): p. 719-725.

22. Asuman, D., C.G. Ackah, and U. Enemark, Inequalities in child immunization coverage in Ghana: evidence from a decomposition analysis. Health economics review, 2018. 8(1): p. 9.

23. Ngugi, A.K., et al., Cohort Profile: The Kaloleni/Rabai Community Health and Demographic Surveillance System. International Journal of Epidemiology, 2019.

24. Government of Kenya, Ministry of Health, and (GoK;MoH). Kenya Master Health Facility List. 2018. 17 May 2018]; Available from: http://kmhfl.health.go.ke.

25. Karuri, J., et al., DHIS2: the tool to improve health data demand and use in Kenya. Journal of Health Informatics in Developing Countries, 2014. 8(1).

26. Maina, J., et al., A spatial database of health facilities managed by the public health sector in sub Saharan Africa. Scientific data, 2019. 6(1): p. 1-8.

27. Ouma, P.O., et al., Access to emergency hospital care provided by the public sector in sub-Saharan Africa in 2015: a geocoded inventory and spatial analysis. The Lancet Global Health, 2018. 6(3): p. e342-e350.

28. Macharia, P.M., et al., Spatial models for the rational allocation of routinely distributed bed nets to public health facilities in Western Kenya. Malaria journal, 2017. 16(1): p. 367. 
29. Noor, A.M., et al., Modelling distances travelled to government health services in Kenya. Tropical Medicine \& International Health, 2006. 11(2): p. 188-196.

30. Ottichilo, W.K., Regional centre for mapping of resources for development (RCMRD): geo-information capacity building activities in Africa. Proceedings of the International Archives of the Photogrammetry, Remote Sensing and Spatial Information Science, 2006. 36(Part 6).

31. Macharia, P.M., et al., Spatial accessibility to basic public health services in South Sudan. Geospatial health, 2017. 12(1): p. 510.

32. Tobler, W., Three presentations on geographical analysis and modeling: National Center for Geographic Information and Analysis. University of California, Santa Barbara, Santa Barbara, CA93106-4060, 1993.

33. Guagliardo, M.F., Spatial accessibility of primary care: concepts, methods and challenges. International journal of health geographics, 2004. 3(1): p. 3.

34. Wang, F., Quantitative methods and applications in GIS. 2006: CRC Press.

35. Molla, Y.B., et al., Best practices in availability, management and use of geospatial data to guide reproductive, maternal, child and adolescent health programmes. BMJ Global Health, 2019. 4(Suppl 5): p. e001406.

36. Aoun, N., H. Matsuda, and M. Sekiyama, Geographical accessibility to healthcare and malnutrition in Rwanda. Social science \& medicine, 2015. 130: p. 135-145.

37. World Health Organization. AccessMod 5. Supporting Universal Health Coverage by modelling physical accessibility to health care 2020 [cited 2020 July]; Available from: https://www.accessmod.org/.

38. Gelman, A., Prior distributions for variance parameters in hierarchical models (comment on article by Browne and Draper). Bayesian analysis, 2006. 1(3): p. 515-534.

39. Government of Kenya, Ministry of Health, and (GoK;MoH), Kenya Health Sector Strategic and Investment Plan(KHSSIP): July 2013-June 2017. 2014: Nairobi,Kenya.

40. Kironji, A.G., et al., Identifying barriers for out of hospital emergency care in low and low-middle income countries: a systematic review. BMC health services research, 2018. 18(1): p. 291.

41. Lankowski, A.J., et al., Impact of geographic and transportation-related barriers on HIV outcomes in sub-Saharan Africa: a systematic review. AIDS and Behavior, 2014. 18(7): p. 1199-1223.

42. Abdulraheem, l., et al., Reasons for incomplete vaccination and factors for missed opportunities among rural Nigerian children. J Public Health Epidemiol, 2011. 3(4): p. 194-203.

43. Ndiritu, M., et al., Immunization coverage and risk factors for failure to immunize within the Expanded Programme on Immunization in Kenya after introduction of new Haemophilus influenzae type $b$ and hepatitis $b$ virus antigens. BMC public health, 2006. 6(1): p. 132.

44. Calhoun, L.M., et al., Determinants and coverage of vaccination in children in western Kenya from a 2003 cross-sectional survey. The American journal of tropical medicine and hygiene, 2014. 90(2): $\mathrm{p}$. 234-241. 
45. Kawakatsu, Y. and S. Honda, Individual-, family-and community-level determinants of full vaccination coverage among children aged 12-23 months in western Kenya. Vaccine, 2012. 30(52): p. 75887593.

46. Kawakatsu, Y., et al., Effects of three interventions and determinants of full vaccination among children aged 12-59 months in Nyanza Province, Kenya. Public Health, 2015. 129(11): p. 1530-1538.

47. Glenton, C., et al., Can lay health workers increase the uptake of childhood immunisation? Systematic review and typology. Tropical Medicine \& International Health, 2011. 16(9): p. 1044-1053.

48. Gachau, S., et al., Does audit and feedback improve the adoption of recommended practices? Evidence from a longitudinal observational study of an emerging clinical network in Kenya. BMJ global health, 2017. 2(4): p. e000468.

49. Schneider, $\mathrm{H}$. and U. Lehmann, From community health workers to community health systems: time to widen the horizon? Health Systems \& Reform, 2016. 2(2): p. 112-118.

50. Owino, L., et al., Factors influencing immunisation coverage in Mathare Valley, Nairobi. East African medical journal, 2009. 86(7).

51. Ogero, M., et al., Examining which clinicians provide admission hospital care in a high mortality setting and their adherence to guidelines: an observational study in 13 hospitals. Archives of Disease in Childhood, 2020.

52. Irimu, G., et al., Tackling health professionals'strikes: an essential part of health system strengthening in Kenya. BMJ global health, 2018. 3(6).

\section{Figures}

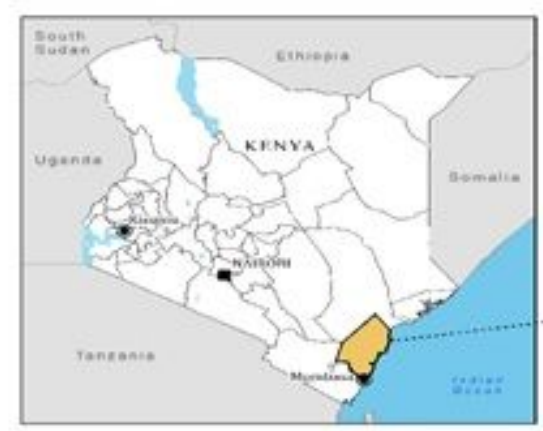

Rabai

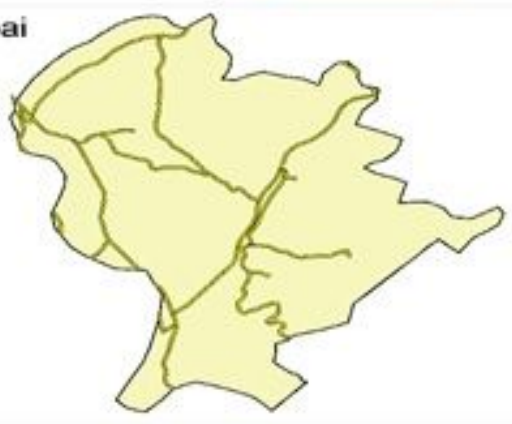

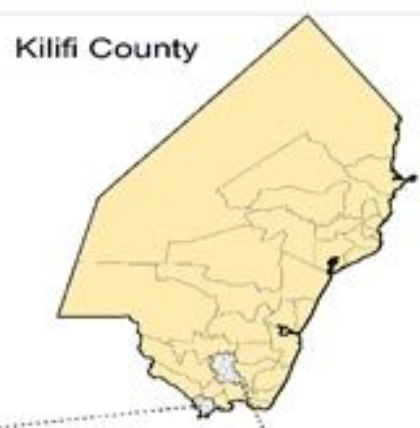

Kaloleni

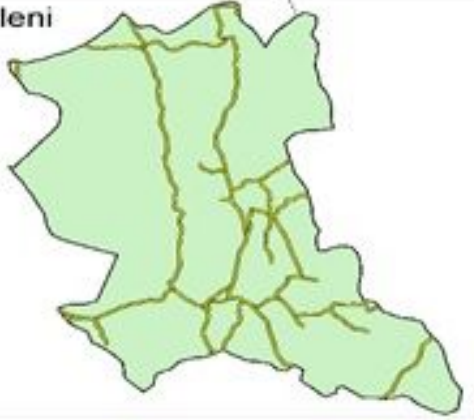

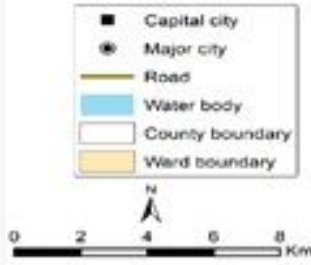

Figure 1 
Map of Kaloleni-Rabai Sub-counties where Community Health Demographic Surveillance System is implemented

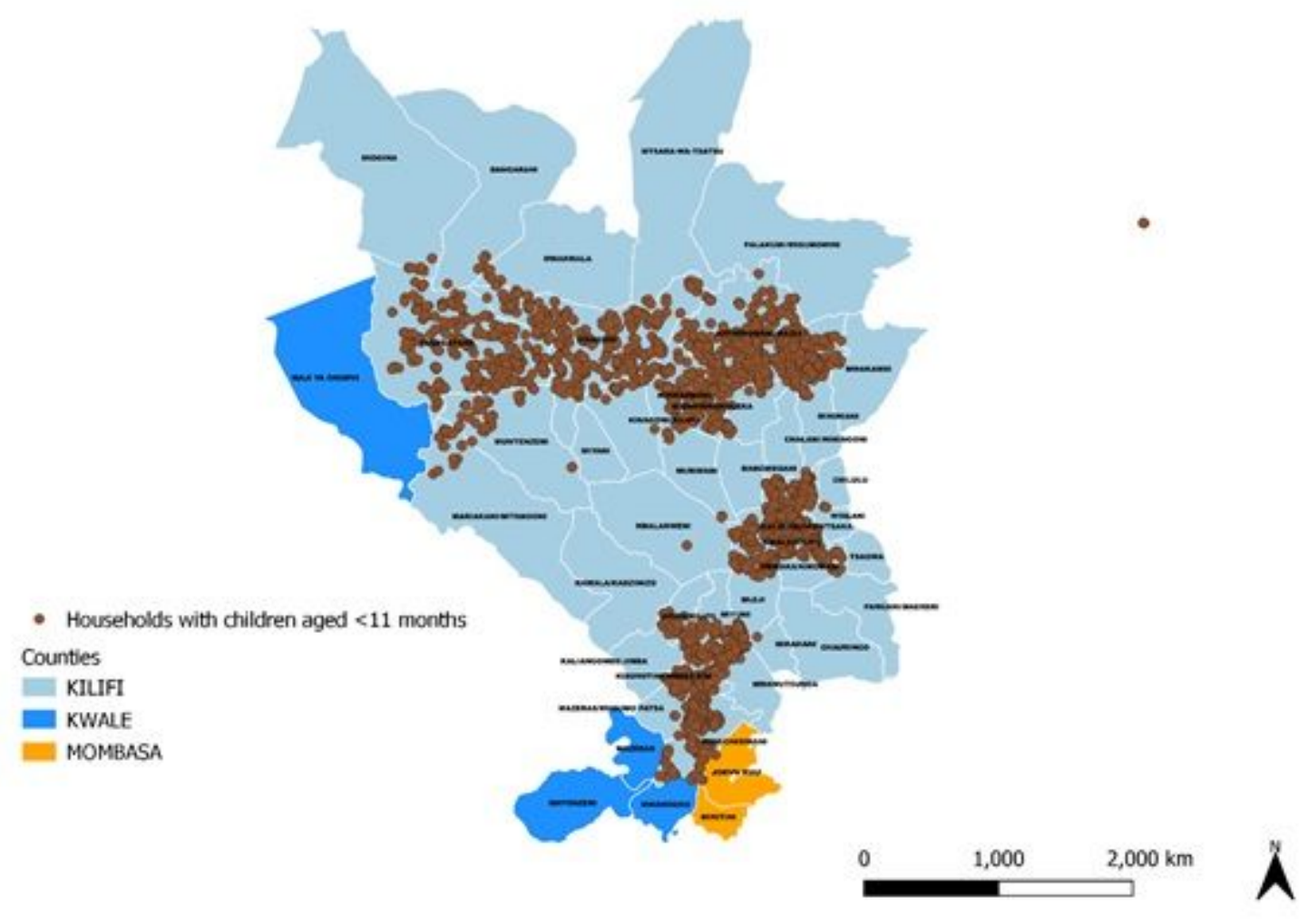

Figure 2

Map showing the distribution of the households with children aged $<11$ months in various sublocations in 3 counties i.e. Kwale, Kilifi and Mombasa. 


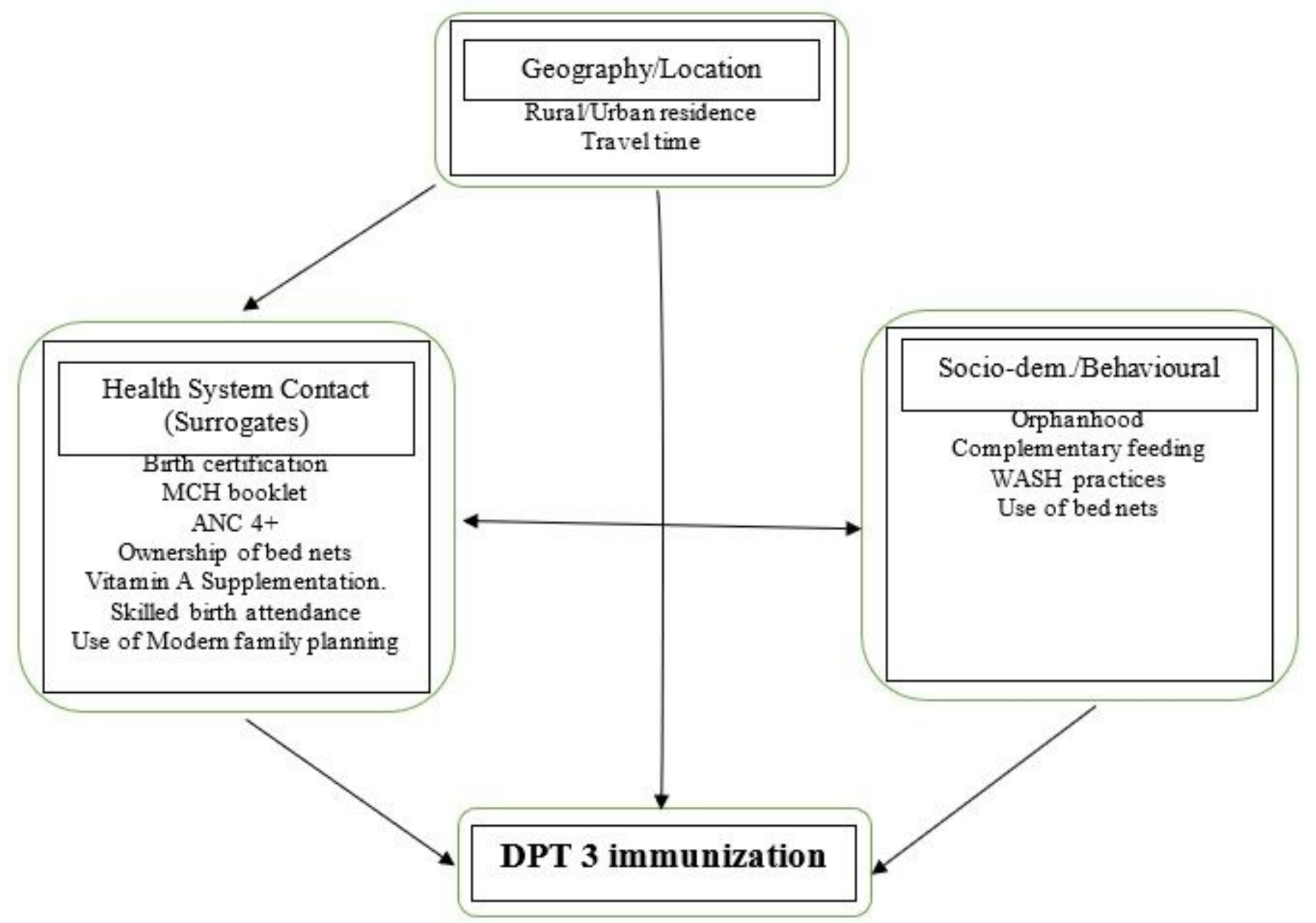

Figure 3

Conceptual framework 


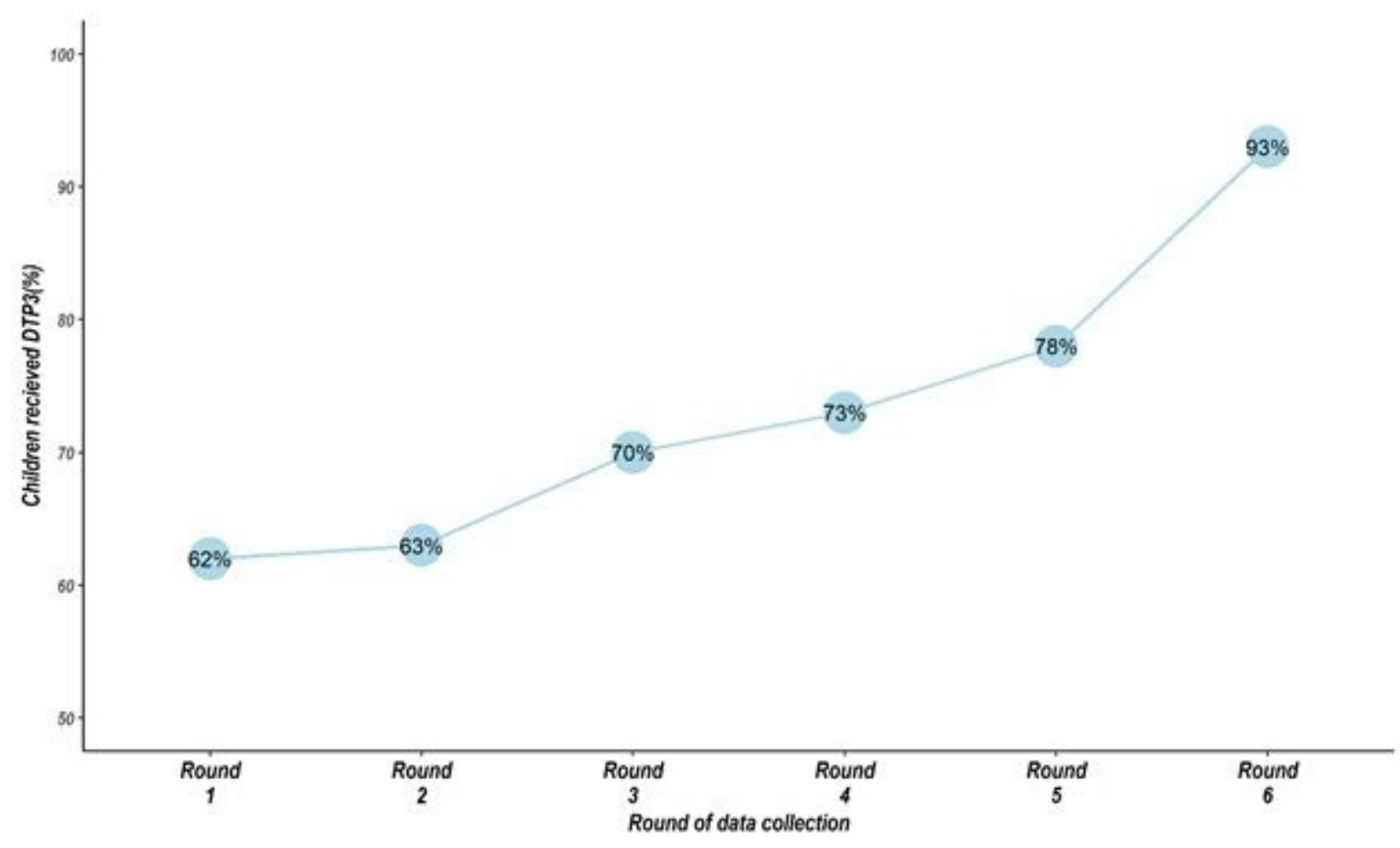

Figure 4

Vaccination coverage over the rounds of data collection in the community demographic surveillance system.

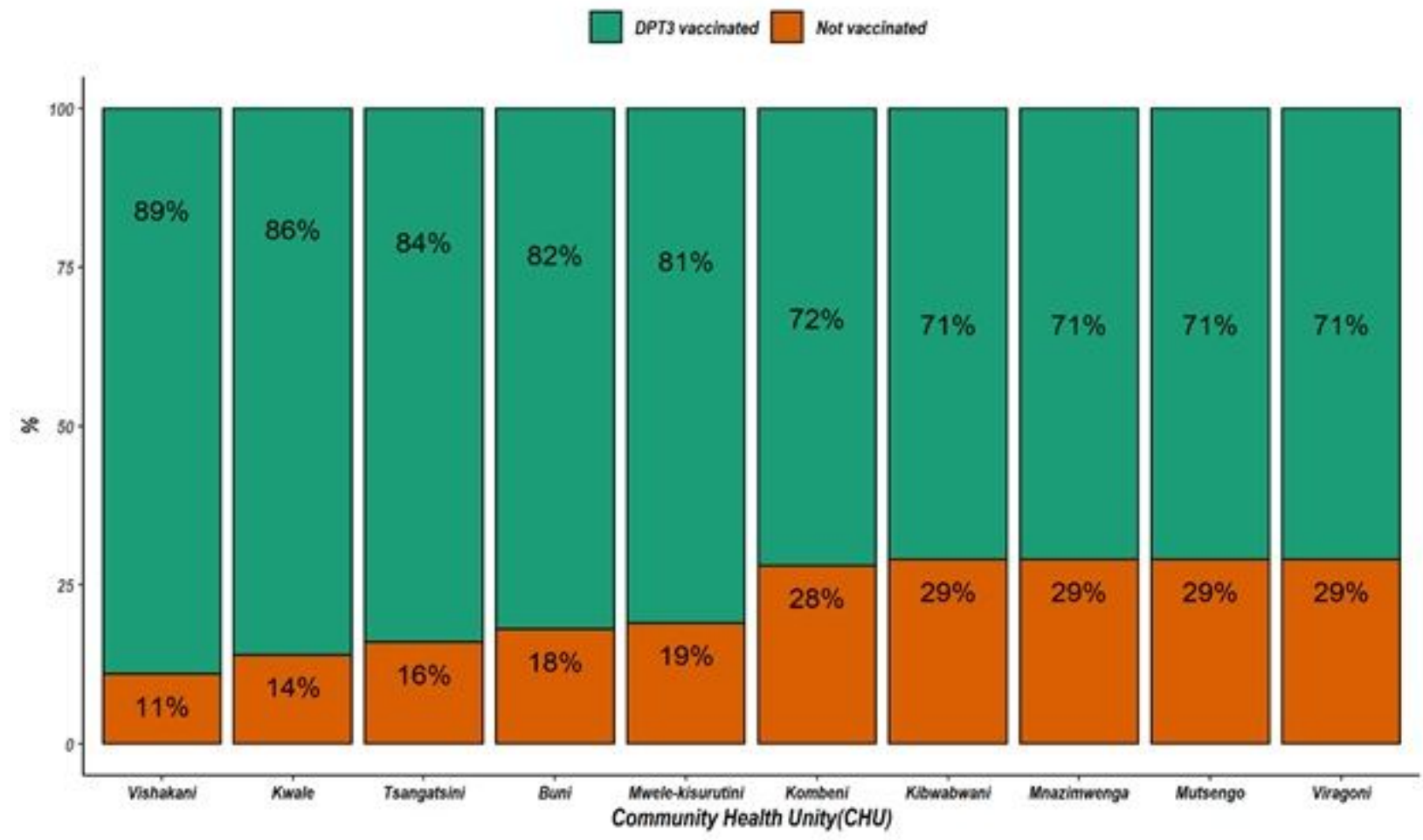

Figure 5

Vaccination coverage across community health units participating demographic surveillance system. 
† Immunising facilities Travel time in minutes

$\square<=20$

$\square>20-40$

$\square>40-60$

- $>60$

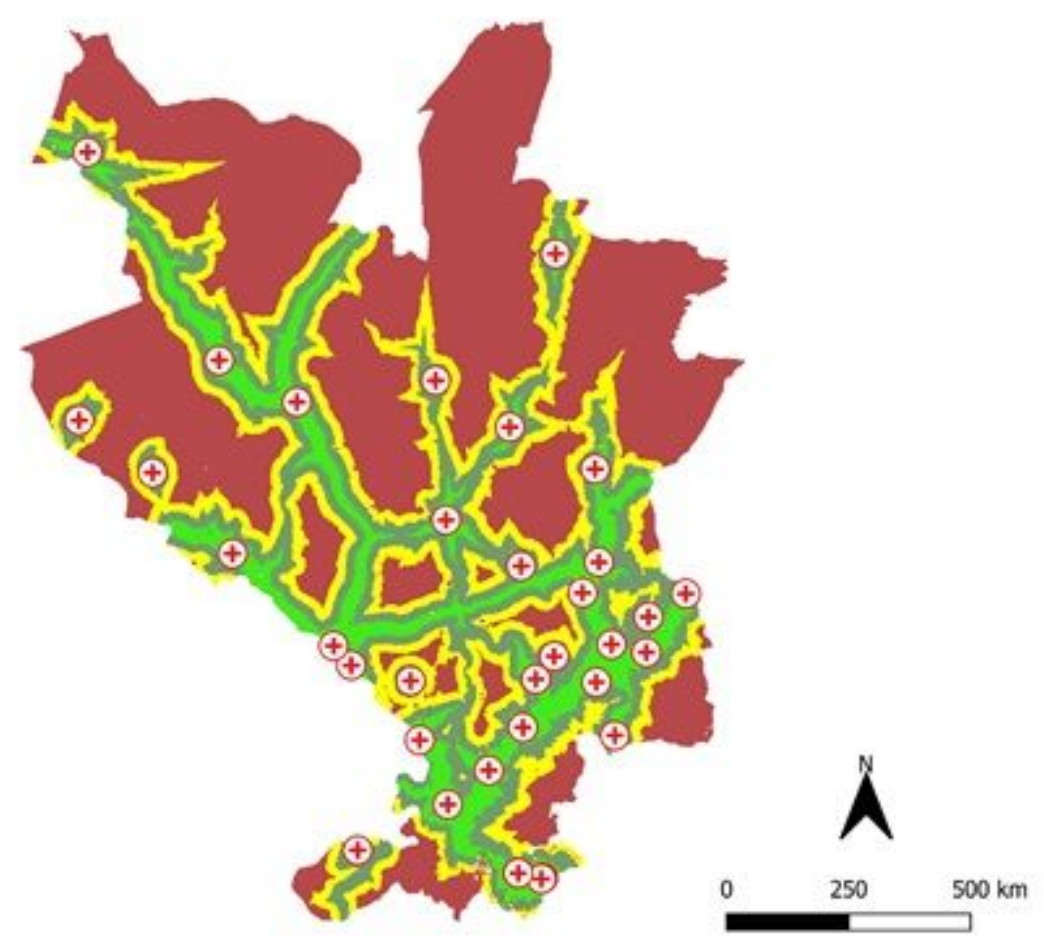

Figure 6

Distribution of mean travel time from each grid $(30 \times 30 \mathrm{~m})$ to the nearest immunising health facility (red cross). The travel time was composite of walking and motorized transport to the nearest immunising health facility in the study area. 